\title{
Non-rigid Multi-modal Medical Image Registration: A Review
}

\author{
Tingting Xu and Ning Wei ${ }^{\star}$ \\ College of Computer and Information Technology, \\ China Three Gorges University, China \\ xtt_ctgu@163.com, \\ weininglz@163.com
}

\begin{abstract}
Medical image registration is an important research topic in image processing field. It plays an important role in future analysis of medical images. Since in that field, the images are always obtained from different modalities and are deformed by non-rigid transform, the registration remains a challenging task for medical images. Many solutions have been put forward currently, but the maturity of these algorithms still remains to be improved. In this survey, we give an overview of these registration methods. We begin by introducing the framework and the elements of which non-rigid registration consists. This is followed by an overview of the classification of non-rigid registration methods, and then a more detailed analysis of the fundamental principle and the research process of the methods is provided. We conclude with a summary and promising future research directions.
\end{abstract}

Key words: Non-rigid registration, Multi-modal image, Mutual information

\section{Introduction}

Medical image registration is a method that looks for a kind of spatial transformation and then puts two images into the corresponding position to achieve area of research full agreement at spatial and anatomic locations. However, the task remains a challenging and active area of research. The difficulty comes from the combination of two frequently encountered characteristics of medical images. The first characteristic is that the transformation model between two images is always including no-rigid deformation. This is caused by patient motion, due to different positioning or breathing level, and pathological change between scans. The second characteristic is that the images are multi-modal images which are obtained from different scan systems. Alignment of multi-modal images helps to relate clinically relevant and complementary information from different scans. For example, it can be used in image guided interventions. In current clinical applications, the registration between computed tomography (CT) and Magnetic resonance imaging (MRI) images is one of the commonly used multi-modal image registrations. By matching the images of the two modes, we can mix the spatial information of high-density organized on bones of the CT images and the spatial information of soft tissues of MRI images and then provide more reliable analysis data for diagnosis.

\footnotetext{
${ }^{\star}$ Ning Wei is the corresponding author.
} 
In the past three decade, many widely cited review papers are published. Some of these papers were only focused on registering photograph taken by ordinary camera or remote sensing. Some of the papers were only focused on registering multi-modal image, with no non-rigid deformation in medical images. However, for passing 10 years non-rigid multi-modal medical image registration becomes a hot topic in image processing field, variance methods are proposed for handling this difficult problem.

In this paper, we summarize these methods which are proposed in high cited ratio papers, classify these methods by the idea behind them. Furthermore, we highlight current research trends in deformation transformation modeling, similarity measurements modeling and provide insights for future.

The paper is structured as follows: section 2 review the basic registration framework and components. This include mathematic description for registration and elementary technicals related to registration. Section 3 reviews most widely used non-rigid transformation models. This include space based models and physical base models. Section 4 reviews the state of the art similarity measurement functions. This include Mutual Information (MI) based measurements, muti-modal invariant descriptor based measurements. We address our discussions and conclusions in section 5 .

\section{Non-rigid registration framework and components}

\subsection{Mathematic description of non-rigid registration}

Given two images $R$ (referencing image) and $F$ (floating image), they are defined in the grid $\Omega$ and mapping to the gray values $r, f=1, \cdots, n$. Then the registration formula can be written as follows:

$$
\hat{T}=\underset{\tau}{\arg \min } S(R, F(\tau))
$$

where $\tau$ represents the transformation space, $S$ expresses the similarity measure. If the two images are perfect aligned, there is $R(x)=F(\tau), x \in \Omega$. The aim of image registration is to find a proper transformation $T$ to make the similarity metric $S$ achieve the maximum.

\subsection{Framework of non-rigid registration}

The framework of non-rigid registration is not absolute, but for a certain type of nonrigid registration method there are certain registration steps. The core task of the current image non-rigid registration research is to determine the image geometric transform space which is supported by the parameter $\tau$ in (1). It is illustrated in Fig. 1 that the whole procedure of the registration, estimates the paprameter $\tau$, is a procedure of iterating.

Firstly, it is showed in Fig. 1, the floating image is first Transformed using an initial parameter $\tau_{n}$, and then compare the effects of alignment with reference image using the Measurement similarity function. As the Floating image is transformed, the Image interpolation methods is needed to interpolate the floating image space into the coordinates of another image space. 


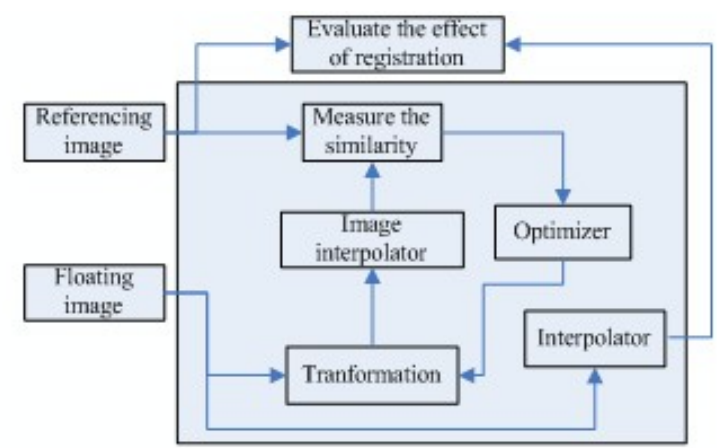

Fig. 1. Framework of non-rigid registration

After measuring the similarity, we need to find another better parameter $\tau$ which can gain more similarity measurement than previous measurement. Therefor, the similarity measurement function $S$ in $(1)$ is treated as cost function with $\tau$ as the multidimensional variable. Since the function $S$ alway have a very complex form, the numerical optimization methods are proposed to compute the the next better parameter $\tau_{n+1}$. Then the newly estimated parameter $\tau_{n+1}$ is take into this iteration procedure again. The whole prcedure will be stopped when the similarity measurement value, or the metrics between two adjacent estimated paprameters $\left\|\tau_{n}-\tau_{n+1}\right\|$ under a given threshold, the finally estimated parameter $\tau$ will also be output at the same time.F

In the following paper, we will summarize the similarity non-rigid transformation models, measurement models, and optimization methods, all these methods are proposed in recently published papers.

\section{Non-rigid Transform Models}

It shows in Fig. 1 that to register two images, the core work is to estimate the parameters which define the model of deformation. The main deformation models can be classified as space transform model and physical based deformation models. We will illustration these models in the following section.

\subsection{Space Model based deformation}

Free Forme Deformation The most commonly used space transformation methods are based on spline function transformation. For the computation efficiency and local deformations with few degrees of freedom. The model is first represented in the work [1], and it is called Free Form Deformation (FFD). The deformation in 2D is given as

$$
T(\mathbf{x})=\sum_{k=1}^{k} \sum_{l=1}^{L} B_{k}(\mu) B_{l}(\nu) d_{k l}
$$


where d denotes displacement, $k=\left\lfloor x / \delta_{x}\right\rfloor, l=\left\lfloor y / \delta_{y}\right\rfloor, \mu=x / \delta_{x}-\left\lfloor x / \delta_{x}\right\rfloor$, and $\nu=y / \delta) y-\left\lfloor y / \delta_{y}\right\rfloor . B_{l}$ represents the $l$ th basis function of the B-spline and $\delta_{x}=\frac{M}{K-1}$ and $\delta_{y}=\frac{N}{L-1}$ denote the contra point spacing. The displacement $\mathbf{d}$ for each control points is the corresponding parameter $\tau$ in (1). The resolution of contra points grid determines the deformation degree of freedom, but also the computational complexity.

Markov radon field based transform The main concept of registration on a Markov random field (MRF) is to set a random discrete displacement label $f_{p}$ to pixel $\mathbf{x}$ for that the maximum posterior probability (MAP). The energy minimization function can be expressed as [2]

$$
E(f)=\sum_{p \in P} S_{p}\left(f_{p}\right)+\sum_{(p, q) \in N} R\left(f_{p}, f_{q}\right),
$$

where $S$ is the measurement, $R$ measures the pairwise cost of assigning $f_{p}$ and $f_{q}$ to two neighboring pixels. Then the problem of registration in (3) can be optimized using some discrete optimization method such as graph cut or belief propagation algorithms.

\subsection{The physical based non-rigid transformation models}

The physical based models can recovery of large deformations, since the model can operate on velocities. But the main drawback of this kind of model is high computation burden. This is the significant reason why the method does't gain high popularity. Most of these methods are expressed basing on the partial differential equation. For the limited space of the paper, we only represents the Elastic models and demons model, other methods such as viscous flow, optical flow are omitted.

Elastic model In elastic model, the non-rigid deformation from the float image to reference image can be modeled as a physical process which is similar to the tensile elastic material. The model is described by the partial differential equation of NavierStokes equation:

$$
\mu \nabla^{2} T+(\mu+\lambda) \nabla(\nabla \cdot T)+\mathbf{F}=0,
$$

where $F$ is the force that drives the registration based on an similarity measurement. [3] firstly proposed to model an image gird as an elastic membrane that is deformed under the influence of two forces that compete until equilibrium is reached.

Diffusion Model In [4], the deformation is modeled by the the diffusion equation:

$$
\nabla T+\mathbf{F}=0
$$

The model was first represented by [5]. It models the object boundaries as membranes and the image was diffused through it under the influence of Demons placed inside the membranes. To registration, we have to estimate the displacements for every pixel, and regularization by (5). 
Demons Model The demons based model was first proposed by the [5], which considers non-parametric non-rigid registration as a diffusion process. It introduced demons that push according to local characteristics of the images in a similar way maxwell did for solving Gibbs paradox. Late on, [6] extended the demons method, and treat the demons as a frame work of alternate optimizing a well-posed minimization criterion. In [6], it models the registration as below global energy:

$$
E(c, s)=\frac{1}{\sigma_{i}^{2}} S(R, F(c))+\frac{1}{\sigma_{x}^{2}} \operatorname{dist}(s, c)^{2}+\frac{1}{\sigma_{T}^{2}} \operatorname{Reg}(s),
$$

where $\sigma_{x}$ accounts for a spatial uncertainty on the correspondences, $c$ is the hidden variable in registration. The demons based method can be view as an alternate optimization of the (6). It alternates between optimizing the first two term and optimizing the last two terms in (6) by using Gaussian smoothing. The advantage of Demons algorithms is that it cover a large variety of constrains, which is determized by $\operatorname{Reg}(s)$ in $(6)$, and computationally efficient with decent accuracy.

In addition to the non-rigid registration methods based on space transformation and physical models introduced above, there are many other registration methods based on the surface of the homologous and these registration methods have been applied to brain images. In practical applications, getting an exact match on cerebral structures has been the bottleneck of brain image registration. Therefore, some researchers consider to use opposite approaches and the basic idea is to use the homologous structure which can be recognized and calculated in the body to generate the deformation.

\section{Similarity mesure function}

\subsection{Mutual information (MI) based models}

MI is derived from information theory and measures the statistical dependency of two random variables. It was first introduced to medical image registration of multi-modal scans by [7] [8]. It is based on the assumption that a lower entropy of the joint intensity distribution corresponds to a better alignment. [9] gives a more detailed review of the MI.

Given two intensity images $R$ and $F$, the mutual information between them is defined as follows:

$$
M I(R, F)=H(R)+H(F)-H(R, F),
$$

where $H(R)$ and $H(F)$ means the information entropy of image $R$ and $F$, and $H(R, F)$ is the joint information entropy of $R$ and $F$. In order to represent how to compute MI in (7), we firstly assume that $p(i)$ is the probability density of gray value which equals to intensity $i$ in image $I(\mathbf{x})$ at all pixel labels $\mathbf{x} \in \Omega$. Secondly, we assume that $p(r, f)$ as the joint probability density between image $R(\mathbf{x})$ and $F(\mathbf{x})$ at the same pixel labels $\mathrm{x} \in \Omega$. Then the MI in (7) can be computed as

$$
M I(R, F)=\sum_{r} \sum_{f} p(r, f) \log \frac{p(r, f)}{p(r) p(f)} .
$$

When the two images reach a consistency in spatial anatomical position, they reach the maximum mutual information. 
normalized mutual information (NMI) The normalized mutual information based on measurement is an extension of mutual information. [10] proved that mutual information depends on the overlap of two images. If the overlaps are too small, similarity measures based on (MI) it is difficult to achieve the ideal effect of registration. In order to solve this problem, [10] proposed to use the normalized mutual information as an index to evaluate registration results. The formula for the normalized mutual information is:

$$
N M I(R, F)=\frac{H(R)+H(F)}{H(R, F)} .
$$

It shows in (7) (8) (9) that an important disadvantage of traditional mutual information based function for image registration is that it does not use the spatial information shared across images. In the presence of image intensity distortions, the MI base methods deteriorate the quality of the alignment, especially in the case of non-rigid registration where the geometric constrains of the transformation are relaxed compared to rigid body alignment. To overcome these disadvatage, various methods include spatial information into the joint and marginal histogram computation. We listed some methods below which are based on this idea.

Conditional mutual information (CMI) [11] introduce a third channel to the joint histogram containing a spatial or regional label. The work is is called conditional mutual information (CMI). The image is subdivided into a number of overlapping region and each intensity pair only contributes to its specific regional histograms. A number of anchor points $\mathbf{X}$ are evenly distributed on the image grid. They evaluate the measure function as $M I(R, F \mid X)$, the mutual information between $R$ and $F$ given a certain spatial distribution $X$.

$$
M I(R, F \mid X)=\sum_{x} p(x) M I(R, F \mid X=x)
$$

In [12], by using this measurement function, the registration method reduces the negative influence of bias field and yield a high registration accuracy. The drawback is the high complexity for computing the 3D histogram. In addition, to separate the image uniformly can't exactly reflect the anatomical spatial structures.

\subsection{Local modality independent descriptor model}

The main idea behind this kind method is to describe the structure of images $R$ and $F$ instead of their different modality. After that the images $R$ and $F$ seems coming from mono-modality imaging. Then the points based registration methods, which are widely used in stereo matching technical, can be used to align the non-rigid images. The size of the descriptor for each pixel is $|R|$. Then the difference measure between $R$ and $F$ at pixel $\mathbf{x}$ can be compute by the sum of absolute difference between descriptors located at that pixel. 
Structual representation In [13], proposed an entropy based structure representation, which can represent two multi-modal images in a similar mono-modal images. After that, square sum difference (SSD) measurement are used across modalities, achieving similar registration accuracy as mutual information for rigid multimodal registration and some synthetic non-rigid images. In [13], the entropy image is obtained by setting each pixel with the newly computed local intensity entropy which is computed by using the histogram formed by the small patch around that pixel. Because the entropy only determined by the relative distribution of the intensities in spite of the value of the intensities, the entropy image only represent the structure of the multimodal image but not the true intensities.

In [13], In order to estimate the histogram exactly, the number of intensity bins should be sufficiently small. This reduce the sensitivity to small intensity changes or make the structure image sensitive to the noise.

Modality independent neighborhood descriptor (MIND) The main aim of the MIND in [14] is to find an image descriptor, which is independent of modality, contrast and noise level of image. The MIND for a pixel $\mathbf{x}$ is defined as

$$
\operatorname{MIND}(R, \mathbf{x}, \mathbf{r})=\frac{1}{n} \exp \left(-\frac{D_{p}(R, \mathbf{x}, \mathbf{x}+\mathbf{r})}{V(R, \mathbf{x})}\right) \quad r \in \mathbf{R},
$$

where $r$ is the spatial extent of the feature region in R. $D_{p}(\cdot)$ is the SSD between the $\mathbf{x}$ and its neighboring $\mathbf{x}+\mathbf{r} . V(\cdot)$ is the estimation of the local variance. For deformation registration, the region $R$ is chosen as a small local neighbor of the $\mathbf{x}$. The measurement function $S$ in 1 is computed by sum of absolute difference (SAD).

Registering CT and MRI clinical images use MIND gained a high accuracy than NMI or CMI. But the MIND is not a rotation invariant descriptor, this drawback result in the low performance when register two image include local rotation deformation. In addition the application of MIND to other multi-modal registration task or anatomical regions need further be investigate.

\section{Conclusions}

In this paper, we reviewed the state of the art non-rigid multi-modal image registration methods. After giving the full view of each component which included in registration, we elaborate the widely used deformation models and the similarity measurement functions. In addition, we showed the main ideas behind these methods and analyzed their advantages and drawbacks.

In conclusion, the deformable registration to multi-modal medical images has so far remained a hot topic and a hard problem. It attracts more interesting from the reachers in the mathematic field, for that many new physics based model such as using NavierStokes equation are proposed to describe deformation. This will provide engineer more tools to solve the larger deformation problem. From the material, we can see that MI are still the main base that many measurement function are based for registration. But now, the state of art paper represented, CMI or structure based methods, are all focus 
on solving the disadvantage of the MI, which is that it ignores the spatial neighborhood. To embed the spatial information in the MI will significantlly improve the registration results.

Along with the more accurate model of the registration, the $S$ and $T$ in (1) will become more complicated. Another big challenge for researches is how to numerically estimate the parameters. One of the scheme for using the discrete optimization method to solve the complexity similarity function is based on MRF discrete labelling, which may allow us to find better way to maximize the function.

\section{Acknowledgment}

This work was supported by the National Natural Science Foundation of China (Grant No. 61202141).

\section{References}

1. D.Rueckert, L. I. Sonoda, C. Hayes and D. L. G. Hill, Nonrigid registration using free-form deformations: Application to breast mr images IEEE Transactions on Medical Imaging, vol. 18, no. 8, pp. 712-721, Aug 1999.

2. M. B. Mattias, P. Heinrich and Mark Jenkinson, Non-rigid image registration through efficient discrete optimization Proc. Medical Image Analysis and Understanding (2011).

3. B. Chaim, Optimal registration of deformed images Ph.D. dissertation, Pennsylvania, Pennsylvania of America, 1 (2012).

4. M. Tim and T. Demetri, Deformable models in medical image analysis: a survey Medical image analysis, vol. 1, no. 2, pp. 91108, (1996).

5. Thirion. jean philippe, Image matching as a diffusion process: an analogy with maxwells demons Medical image analysis, vol. 2, no. 3, pp. 243260, (1998).

6. A. P. N. A. Tom Vercauterena, Xavier Pennecb, Diffeomorphic demons: efficient nonparametric image registration Neuroimage, vol. 45, no. 1, pp. S61S72, Mar (2009).

7. M. Frederik, C. Andre, V. Dirk, M. Guy, and S. Paul, Multimodality image registration by maximization of mutual information IEEE Transactions on Medical Imaging, vol. 16, no. 2, (1997).

8. V. Paul and W. I. W. M, Alignment by maximization of mutual information International Journal of Computer Vision, vol. 24, (1997).

9. Pluim Josien P W and Maintz J B Aatoine and Viergever Max A, Mutual-information-based registration of medical images: a survey IEEE Transactions on Medical Imaging, vol. 22, no. 8, pp. 9861004, (2003).

10. S. Colin, H. Derek, and H. David, An overlap invariant entropy measure of 3 d medical image alignment Pattern Recognition, vol. 32, pp. 7186, (1999).

11. S. C. et al, Deformation-based mapping of volume change from serial brain mri in the presence of local tissue contrast change IEEE Transactions on Medical Imaging, vol. 25, (2006).

12. L. Dirk, S. Pieter, M. Frederik, V. Dirk, and S. Paul, Nonrigid image registration using conditional mutual information IEEE Transactions on Medical Imaging, vol. 29, no. 1, pp. 1929, (2010).

13. W. Christian and N. Nassir, Entropy and laplacian images: structural representations for multi-modal registration Medical Image Analysis, vol. 16, no. 1, pp. 117, Jan (2012).

14. M. B. Mattias P. Heinrich, Mark Jenkinson, Mind: Modality independent neighbourhood descrptor for multi-modal deformableregistration Medical Image Analysis, vol. 16, no. 7, pp. 14231435, Oct (2012). 